\title{
Influence du rapport calcium/phosphore de la ration sur la calcémie, la phosphatémie et la magnésémie de la lapine en gestation
}

\author{
M Assane, GP Gongnet, A Coulibaly, A Sere \\ École inter-états des sciences et médecine vétérinaires, BP 5077, Dakar, Sénégal
}

(Reçu le 16 novembre 1992; accepté le 16 mars 1993)

\begin{abstract}
Résumé - Seize lapines de souche Thiès du Sénégal ont été réparties en 2 lots de 8 pour étudier I'influence de deux rapports $\mathrm{Ca} / \mathrm{P}$ de la ration $(1,0$ et 2,1$)$ sur la calcémie, la phosphatémie et la magnésémie au cours de la gestation. Les résultats obtenus ont montré qu'avant la saillie et durant la première semaine de la gestation la calcémie des lapines dont le rapport $\mathrm{Ca} / \mathrm{P}$ de la ration est de 2,1 est significativement plus élevée. Au cours des 15 derniers jours de la gestation la calcémie des 2 groupes d'animaux a baissé de manière significative. La phosphatémie et la magnésémie ne sont pas significativement différentes entre les 2 lots de lapines jusqu'à la deuxième semaine de la gestation. Mais en fin de gestation, alors que la phosphatémie et la magnésémie des lapines dont le rapport $\mathrm{Ca} / P$ de l'aliment est de 2,1 étaient très abaissées, celles des animaux du second lot $(\mathrm{Ca} / P=$ $1,0)$ n'avaient pas varié.
\end{abstract}

calcémie / phosphatémie / magnésémie / rapport Ca:P / gestation

Summary - Effect of calcium/phosphorus ratio on plasma calcium, inorganic phosphorus and magnesium levels during pregnancy in the rabbit. Sixteen Thies strain rabbits were used to study the effect of the calcium/phosphorus ratio on plasma calcium, inorganic phosphorus and magnesium levels during pregnancy. Animals were allocated in 2 groups of 8: one receiving a diet with a CalP ratio of 1:0 and the other a diet with $C a / P$ ratio of $2: 1$. Water was given ad libitum for all rabbits. Results obtained showed that $\mathrm{Ca}$ blood level was significantly higher in rabbits receiving the diet with Ca/P ratio of 2:1 before covering and during the first week of pregnancy. During the last $15 d$ of pregnancy, Ca blood level decreased significantly in the 2 groups of animals.

Plasma inorganic phosphorus and magnesium levels were not significantly different in the 2 groups of rabbits until the 2 nd wk of pregnancy. But at the end of pregnancy, while phosphatemia and magnesemia decreased in rabbits fed a high Ca/P ratio, in the other group these parameters remained unchanged.

calcemia / phosphatemia / magnesemia / Ca:P ratio / pregnancy 


\section{INTRODUCTION}

Graham et Porter (1971) sur des lapines blanches de Nouvelle Zélande et Barlet (1980) sur des lapines fauves de Bourgogne ont remarqué, avec des aliments commerciaux dont le rapport calcium/ phosphore est d'environ 1,8 , que la $2^{\mathrm{e}}$ moitié de la gestation se traduit par une chute de la calcémie et de la phosphatémie. Or, Coulibaly (1992) avait observé chez la lapine souche "Thiès" du Sénégal qu'avec un rapport phosphocalcique de la ration de 2,1 , le bilan du phosphore devient négatif aussi bien avant la saillie que pendant la gestation, alors qu'avec un rapport calcium/phosphore de 1,0 le bilan de $\mathrm{Ca}$ et $\mathrm{P}$ est toujours positif. Nous nous sommes alors proposés d'étudier les variations physiologiques de la calcémie, de la phosphatémie et de la magnésémie chez cette race de lapins en fonction du niveau d'apport en calcium.

\section{MATÉRIEL ET MÉTHODES}

L'expérience a porté sur 16 lapines adultes de souches "Thiès" du Sénégal, de poids variant entre 2,5 et $3 \mathrm{~kg}$. Elles ont été placées individuellement dans des cages à métabolisme.

Après une semaine de régime commun, les animaux ont été répartis en 2 lots de 8 : un lot $A$ dont le rapport $\mathrm{Ca} / \mathrm{P}$ de la ration est de 1,0 et un lot $\mathrm{B}$ recevant l'aliment à rapport $\mathrm{Ca} / \mathrm{P}$ de 2,1 . Ces différents rapports ont été obtenus en modifiant la teneur de l'aliment en calcium (tableau I).

Chaque lapine a reçu $150 \mathrm{~g}$ d'aliment/j distribués en 2 fois : $75 \mathrm{~g}$ à $8 \mathrm{~h}$ et $75 \mathrm{~g}$ à $18 \mathrm{~h}$. L'eau leur a été donnée à volonté.

Après une semaine d'adaptation aux nouveaux régimes, toutes les lapines ont été saillies.

Pour la conduite de l'expérimentation, 6 prélèvements de sang ont été effectués : un prélèvement témoin avant la saillie et 4 autres respectivement à la première, deuxième, troisième et quatrième semaine de la gestation, et un dernier $24 \mathrm{~h}$ après la mise bas.
Le sang a été prélevé par ponction de la veine saphène externe. Après centrifugation, le plasma a été congelé jusqu'à l'analyse. Le dosage de $\mathrm{Ca}, \mathrm{P}$ et $\mathrm{Mg}$ plasmatiques a été fait par colorimétrie selon les méthodes décrites par Skeggs et Hochstrasser (1964) et par Audigie et al (1983).

Les résultats sont présentés sous forme de moyennes \pm écart type. Les moyennes intra et inter-lots ont été statistiquement comparées par analyse de variance suivant le test de Fischer. Les valeurs de $P<0,05$ ont été considérées comme significatives.

\section{RÉSULTATS}

Les résultats (moyennes \pm écart type) sont portés dans le tableau II.

Avant la gestation, les différences entre les 2 lots d'animaux ne sont significatives $(P<0,01)$ que pour la calcémie : donc la

Tableau I. Composition des aliments.

Composants (\%)

Aliment Aliment

A $B$

$\begin{array}{lrr}\text { Maïs concassé } & 42,42 & 41,76 \\ \text { Sorgho } & 17,01 & 16,75 \\ \text { Tourteau d'arachide } & 11,26 & 11,09 \\ \text { Farine de poisson } & 5,96 & 5,87 \\ \text { Fanes d'arachide } & 20,32 & 20,01 \\ \text { Huile d'arachide } & 1,92 & 1,89 \\ \text { Phosphate bicalcique } & 1,08 & 1,06 \\ \text { Carbonate de calcium } & - & 1,54 \\ & & \\ \text { Composition analytique } & & \\ \text { Matières sèches (\% MF) } & 89,45 & 89,43 \\ \text { Matières protéiques (\% MS) } & 17,17 & 16,78 \\ \text { Matières minérales (\% MS) } & 5,79 & 5,88 \\ \text { Matières grasses (\% MS) } & 9,53 & 8,46 \\ \text { Matières cellullosiques (\% MS) } & 9,25 & 9,13 \\ \text { Calcium (\% MS) } & 0,52 & 0,83 \\ \text { Phosphore (\% MS) } & 0,51 & 0,39 \\ \text { Ca/P } & 1,01 & 2,12\end{array}$

MF : matière fraîche; MS : matière sèche. 
Tableau II. Influence du rapport Ca/P de la ration sur la calcémie, la phosphatémie et la magnésémie de la lapine en gestation.

\begin{tabular}{|c|c|c|c|c|c|c|}
\hline \multirow{2}{*}{$\begin{array}{l}\text { Lots } \\
\text { d'animaux }\end{array}$} & \multicolumn{6}{|c|}{ Stade de gestation (sem) } \\
\hline & $0^{*}$ & 1 & 2 & 3 & 4 & $5^{\star}$ \\
\hline \multicolumn{7}{|c|}{ Calcémie $(m g / l)$} \\
\hline $\operatorname{Lot} A(n=8)$ & $123,0^{\mathrm{a}} \pm 4,4$ & $132,5^{a} \pm 6,4$ & $135,8^{a} \pm 3,7$ & $120,0^{b} \pm 7,9$ & $119,1^{b} \pm 6,8$ & $111,0^{c} \pm 3,1$ \\
\hline Lot $\mathrm{B}(n=8)$ & $145,0^{a} \pm 5,9$ & $160,0^{b} \pm 9,6$ & $138,8^{a} \pm 2,0$ & $124,5^{c} \pm 5,8$ & $122,5^{c} \pm 8,0$ & $114,0^{d} \pm 6,8$ \\
\hline $\begin{array}{l}\text { Différence } \\
\text { inter-lots }\end{array}$ & $\mathrm{S}^{\star \star}$ & $S^{* *}$ & NS & NS & NS & NS \\
\hline \multicolumn{7}{|c|}{ Phosphatémie (mg/l) } \\
\hline $\operatorname{Lot} A(n=8)$ & $38,5^{a} \pm 5,6$ & $40,2^{a} \pm 5,4$ & $42,2^{a} \pm 5,7$ & $39,5^{a} \pm 4,9$ & $41,5^{a} \pm 5,2$ & $40,7^{a} \pm 7,0$ \\
\hline Lot $\mathrm{B}(n=8)$ & $43,0^{a} \pm 5,5$ & $45,5^{a} \pm 5,6$ & $41,5^{a} \pm 6,4$ & $38,3^{a} \pm 6,4$ & $29,7^{b} \pm 3,8$ & $25,0^{b} \pm 5,8$ \\
\hline $\begin{array}{l}\text { Différence } \\
\text { inter-lots }\end{array}$ & NS & NS & NS & NS & $S^{* *}$ & $\mathrm{~S}^{* *}$ \\
\hline \multicolumn{7}{|c|}{ Magnésémie $(m g / l)$} \\
\hline Lot $\mathrm{A}(n=8)$ & $28,0^{a} \pm 5,0$ & $26,5^{a} \pm 5,8$ & $31,3^{a} \pm 5,6$ & $27,3^{a} \pm 4,3$ & $32,7^{a} \pm 2,6$ & $26,6^{\mathrm{a}}=\uparrow, 5$ \\
\hline Lot $\mathrm{B}(n=8)$ & $31,5^{\mathrm{a}} \pm 5,2$ & $36,7^{b} \pm 5,3$ & $27,3^{a} \pm 2,6$ & $23,5 c \pm 5,5$ & $22,0^{c} \pm 5,5$ & $19,5^{c} \pm 3.5$ \\
\hline $\begin{array}{l}\text { Différence } \\
\text { inter-lots }\end{array}$ & NS & $S^{\star \star}$ & NS & $\mathrm{S}^{*}$ & $S^{+*}$ & $S^{+*}$ \\
\hline
\end{tabular}

Lot $A$ : rapport $C a / P=1,0 ;$ lot $B:$ rapport $C a / P=2,1$. Dans un même lot, les valeurs de calcémie, phosphatémie et magnésémie qui portent des lettres différentes sont significativement différentes $(P<0,05)$. $0^{*}$ : avant la saillie; $5^{*}$ : $24 \mathrm{~h}$ après la mise base ; $S^{*}:$ différence significative $P<0,05 ; S^{*}:$ différence significative $P<0,01 ;$ NS : différence non significative.

phosphatémie et la magnésémie des lapines recevant l'aliment $B$ ne sont pas supérieures à celles des lapines recevant I'aliment A.

Pendant la gestation, la calcémie des lapines du lot $B$ demeure significativement plus élevée que celle du lot $A(P<0,01)$ jusqu'à la $2^{e}$ semaine de la gestation; audelà, il n'y a aucune différence significative entre les 2 lots. Dans les 2 cas on observe une chute de la calcémie en fin de gestation et $24 \mathrm{~h}$ après la mise bas $(P<0,01)$. Pour le lot $A$, la calcémie passe de $123 \pm$ $4,4 \mathrm{mg} / \mathrm{l}$ avant la saillie à $111 \pm 3,1 \mathrm{mg} / \mathrm{l}$ $24 \mathrm{~h}$ après la mise bas et pour le lot $\mathrm{B}$ de $145 \pm 5,9 \mathrm{mg} / \mathrm{l}$ à $114 \pm 6,8 \mathrm{mg} / \mathrm{l}$.
En fin de gestation, la phosphatémie des lapines du lot $B$ devient significativement plus faible que celle du lot $A(P<$ $0,01)$. Par ailleurs, alors que la concentration plasmatique de $P$ ne varie pas de manière significative au cours de la gestation chez le lot $A$, chez le lot $B$, pendant cette même période, on observe une chute progressive de ce paramètre sanguin : dans ce groupe la phosphatémie passe de 45,5 $\pm 5,6 \mathrm{mg} / \mathrm{l}$ à une semaine de la gestation à $29,7 \pm 3,8 \mathrm{mg} / \mathrm{l}$ en fin de gestation et $25 \pm$ $5,8 \mathrm{mg} / \mathrm{l}$ post-partum.

Chez les lapines du lot $A$, la magnésémie, bien que fluctuante, ne varie pas de manière significative durant la gestation. 
Par contre, chez les animaux du lot B, la concentration sanguine de $\mathrm{Mg}$, après un pic au cours de la $1^{\text {re }}$ semaine de gestation, chute de manière significative $(P<$ 0,01 ) à partir de la $3^{\mathrm{e}}$ semaine; elle passe de $31,5 \pm 5,2 \mathrm{mg} / \mathrm{l}$ avant la saillie à 19,5 \pm $3,5 \mathrm{mg} / \mathrm{l} 24 \mathrm{~h}$ après la mise bas.

\section{DISCUSSION}

Chez la lapine souche Thiès du Sénégal, la calcémie (en moyenne 123,0 $\pm 5,4 \mathrm{mg} / \mathrm{l}$ pour un régime dont le rapport $\mathrm{Ca} / \mathrm{P}$ est de 1,$0 ; 145,0 \pm 5,9 \mathrm{mg} / \mathrm{l}$ pour une ration à rapport phosphocalcique de 2,1) est plus élevée que celle rapportée chez les autres espèces animales dont la calcémie est en moyenne de $100 \mathrm{mg} / \mathrm{l}$. Par ailleurs, ces résultats montrent que la calcémie de la lapine est fonction du niveau d'apport en $\mathrm{Ca}$, ce qui laisse supposer que les mécanismes régulateurs de la calcémie chez cette espèce sont moins efficaces que chez les autres espèces. Cette hypothèse nous semble d'autant plus plausible que la valeur de 123,0 $\pm 5,4 \mathrm{mg} / \mathrm{l}$ obtenue avec un rapport $\mathrm{Ca} / \mathrm{P}$ de 1,0 correspond à celle obtenue par Barlet (1980) chez la lapine fauve de Bourgogne $(123,7 \pm 3,8 \mathrm{mg} / \mathrm{l})$, avec un rapport $\mathrm{Ca} / \mathrm{P}$ de 1,8 . De même, la calcémie de $145 \mathrm{mg} / \mathrm{l}$ que nous avons obtenue avec le rapport $\mathrm{Ca} / \mathrm{P}$ de 2 est supérieure à celle obtenue par Barlet (1980) et par Agkün et Rudman (1969), les valeurs obtenues par ces 2 groupes d'auteurs étant elles-mêmes différentes.

La comparaison de la calcémie des 2 lots de lapines montre qu'une augmentation de la teneur de la ration en calcium se traduit par une augmentation de la calcémie. Ainsi chez le lapin, contrairement aux autres espèces animales, il y a une relation étroite entre la calcémie et la teneur de l'aliment en calcium. Ces observations sont en accord avec celles de Chapin et
Smith (1967) et de Besançon et Lebas (1969).

Dans les 2 lots de lapines, les valeurs de la phosphatémie (lot $A=38,5 \pm 5,6 \mathrm{mg} /$; lot $B=43,0 \pm 5,5 \mathrm{mg} / \mathrm{l}$ ) sont inférieures à celles obtenues par Barlet (1980) chez la lapine fauve de Bourgogne $(61,0 \pm 4,0 \mathrm{mg} / \mathrm{l})$. Par contre, la magnésémie de la lapine souche Thiès du Sénégal $(28,0 \pm 5,0 \mathrm{mg} / \mathrm{l}$ pour le lot $A ; 31,5 \pm 5,2 \mathrm{mg} / \mathrm{l}$ pour le lot $B$ ) est plus élevée que celle rapportée par Barlet (1980). Les différences entre nos résultats et ceux de l'auteur précité pourraient être liées à la race, aux conditions climatiques ou au dosage des minéraux. Cependant, l'égalité des valeurs de la phosphatémie entre nos 2 lots d'animaux est conforme à ce qui est rapporté pour d'autres espèces animales (Howard, 1989).

La bibliographie reste muette quant à l'influence du rapport $\mathrm{Ca} / \mathrm{P}$ de la ration sur la calcémie, la phosphatémie et la magnésémie chez la lapine en gestation. Mais Barlet (1980), sur des lapines fauves de Bourgogne, observe une chute de la calcémie et de la phosphatémie dans les 20 derniers jours de la gestation et les 20 premiers jours de la lactation, alors que la magnésémie ne varie pas au cours de ces stades physiologiques. Nos résultats sont conformes à ceux de Barlet (1980) en ce qui concerne la calcémie pour les 2 rapports $\mathrm{Ca} / \mathrm{P}$, la phosphatémie pour le rapport $\mathrm{Ca} / \mathrm{P}$ de 2,1 et la magnésémie pour le rapport $\mathrm{Ca} / \mathrm{P}$ de 1,0 . Par contre, chez la lapine, contrairement à ce qui a été montré chez la vache (Boda and Cole, 1954; Brochart, 1964), la teneur en calcium de la ration distribuée au cours de la gestation ne semble pas avoir d'influence sur l'hypocalcémie, alors qu'elle conditionne les variations simultanées de la phosphatémie et de la magnésémie.

La chute de la calcémie chez la femelle mammifère en fin de gestation a été rap- 
portée par plusieurs auteurs (Payne, 1966; Braithwaite et al, 1970; Twardock et al, 1971; Sigurdsson, 1988; Assane et al, 1989). L'hypocalcémie qui intervient chez la lapine avant le part peut être la conséquence d'une mobilisation importante de Ca maternel au profit des fœtus, comme l'ont suggéré de nombreux auteurs chez d'autres espèces animales (Braithwaite et al, 1970; Twardock et al, 1971; Sigurdsson, 1988). Une autre cause possible de cette hypocalcémie prépartum peut être la stimulation œstrogénique pendant cette période, comme l'ont montré les travaux de Payne (1966) chez la chèvre.

En ce qui concerne la phosphatémie, nous avons constaté qu'elle ne varie pas au cours de la gestation lorsque le rapport $\mathrm{Ca} / \mathrm{P}$ de la ration est de 1 , alors qu'avec un rapport $\mathrm{Ca} / \mathrm{P}$ de 2 la concentration sanguine de ce minéral chute en fin de gestation. Cette disparité des résultats doit toutefois tenir compte des difficultés que présente le dosage du phosphore inorganique. En effet, la fraction minérale du phosphore sanguin est très labile, si bien que différentes conditions physiologiques peuvent modifier notablement son taux ( $\mathrm{Va}$ lade, 1981).

Pour ce qui est de la magnésémie, très peu de données sont disponibles à propos de sa régulation chez la lapine. Mais la chute de la magnésémie que nous avons observée chez les lapines en fin de gestation lorsqu'on enrichit l'aliment en $\mathrm{Ca}$, peut trouver une explication à travers les résultats obtenus par Aikawa et Bruns (1960). En effet, ces auteurs rapportent que chez la lapine en fin de gestation, il y a une augmentation du prélèvement de Mg maternel par les fœtus.

\section{CONCLUSION}

Nos résultats démontrent que, quelle que soit la teneur de l'aliment en calcium, il se produit une chute de la calcémie chez la lapine en fin de gestation. Par contre, l'évolution de la phosphatémie et de la magnésémie durant cette période dépend du rapport phosphocalcique de la ration.

\section{RÉFÉRENCES}

Agkün S, Rudman D (1969) Relationships between mobilization of free fatty acids from adipose tissue, and the concentrations of calcium in the extracellular fluid and in the tissue. Endocrinology 84, 926-930

Aikawa JK, Bruns PD (1966) Placental transfer and fetal tissue uptake of $\mathrm{Mg}^{28}$ in the rabbit. Proc Soc Exp Biol Med 105, 95-98

Assane M, Sere A, Djimrao $S$, Sow R, Ba AC, Gaye $O$ (1989) Variations physiologiques de la calcémie et de la phosphatémie chez la brebis Peul du Sahel en gestation. Sci Vét Méd Comp 91, 263-271

Audigie C, Dupont C, Zonszain F (1983) Principe des méthodes d'analyses biochimiques. Doin, Paris, tome 2, $122 \mathrm{p}$

Barlet JP (1969) Variation de la calcémie et de la phosphatémie chez la vache laitière au moment du vêlage : rôle probable de la calcitonine dans l'étiologie du syndrome. Rech Vét 2, 93-100

Barlet JP (1980) Plasma calcium, inorganic phosphorus and magnesium levels in pregnant and lactating rabbits. Reprod Nutr Dev 20,647-651

Besançon P, Lebas F (1969) Utilisation digestive réelle et rétention du calcium par le lapin en croissance recevant un régime riche en calcium et phosphore. Ann Zootech 18, 437-443

Boda JM, Cole HH (1954) The influence of dietary calcium and phosphorus on the incidence of milk fever in dairy cattle. J Dairy Sci $37,360-372$

Braithwaite GD, Glascock RF, Riazuddin S (1970) Calcium metabolism in pregnant ewes. Br J Nutr 24, 661-670

Brochart M (1964) La prévention de l'hypocalcémie vitulaire. Ann Zootech 13, 63-69

Chapin RE, Smith SE (1967) The calcium tolerance of growing and reproducing rabbits. Cornell Vet $57,480-491$ 
Coulibaly A (1992) Contribution à l'étude de l'influence du rapport calcium/phosphore alimentaire sur le métabolisme phosphocalcique et sur certains paramètres de reproduction chez le lapin. Thèse Med Vet Dakar, $n^{\circ} 6$

Graham RW, Porter GP (1971) Fetal-maternal plasma calcium relationships in the rabbit. Q J Exp Physiol 56, 160-168

Howard G (1989) Calcium metabolism and physiology of bone. Handbk Physiol, 21th ed, sect 16, 1461-1479

Payne JM (1966) Survey of milk fever in goats. Vet Rec 78, 31-33

Sigurdsson H (1988) The effect of flock, number of fœtuses and age on some biochemical blood constituents in ewes in late pregnancy under field conditions. J Vet Med 35, 417-423

Skeggs LT, Hochstrasser H (1964) Multiple automatic sequential analysis. Clin Chem 10 , 918-936

Twardock AR, Symonas HW, Samson BR (1971) Effect of the number of fœtuses present in utero on the transfer rates of calcium and phosphorus accross the placenta of the ewe. Int Atom Energy Ag Vienna 312, 2/12, $139 \mathrm{p}$

Valade $G$ (1981) Étude de la variation de certains paramètres enzymatiques et minéraux durant la gestation et les 2 premiers mois de la lactation chez la vache laitière. Thèse doctorat vét, Toulouse, $n^{\circ} 76$ 